\title{
Gold Futures Returns and Realized Moments: A Forecasting Experiment Using a Quantile-Boosting Approach
}

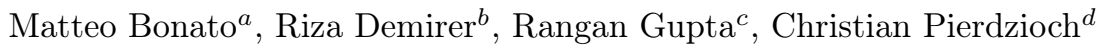

June 2016

\begin{abstract}
This paper proposes an iterative model-building approach known as quantile boosting to trace out the predictive value of realized volatility and skewness for gold futures returns. Controlling for several widely studied market- and sentiment-based variables, we examine the predictive value of realized moments across alternative forecast horizons and across the quantiles of the conditional distribution of gold futures returns. We find that the realized moments often significantly improve the predictive value of the estimated forecasting models at intermediate forecast horizons and across quantiles representing distressed market conditions. We argue that realized moments carry information that reflects investors' tradeoff between diversification and skewed payoffs, particularly during periods of market stress, which may be especially relevant for gold as the traditional accepted safe haven.
\end{abstract}

JEL classification: C22; C53; Q02

Keywords: Gold futures returns; Realized volatility; Realized skewness; Forecasting; Quantile boosting

\section{Addresses:}

${ }^{a}$ Department of Economics and Econometrics, University of Johannesburg, Auckland Park, Johannesburg, South Africa; E-mail: mbonato@uj.ac.za.

${ }^{b}$ Department of Economics \& Finance, Southern Illinois University Edwardsville, Edwardsville, IL 62026-1102, USA; E-mail address: rdemire@siue.edu.

c Corresponding author. Department of Economics, University of Pretoria, Pretoria, 0002, South Africa; E-mail address: rangan.gupta@up.ac.za.

${ }^{d}$ Department of Economics, Helmut Schmidt University, Holstenhofweg 85, P.O.B. 700822, 22008 Hamburg, Germany; Email address: c.pierdzioch@hsu-hh.de. 


\section{Introduction}

The financial market crises and prolonged uncertainty surrounding global economic fundamentals have drawn the attention of researchers towards the dynamics of gold returns as the traditionally accepted safe haven. While the literature has promoted gold as an investment asset due to its low level of correlation with equity indices (Hillier et al., 2006) and its counter-cyclical reaction to unexpected macroeconomic news (Roache and Rossi, 2009), other studies have focused on the determinants of gold returns in the context of its value as a hedge and/or diversifier for investors (Baur and Lucey, 2010; Ciner et al., 2013). Classic determinants of gold returns that have been discussed in earlier literature include stock market returns, exchange-rate movements, oil-price fluctuations, and interest rates (see, for example, Hammoudeh and Yuan, 2008; Pukthuanthong and Roll, 2011; Reboredo 2013a,b; Pierdzioch et al. 2014; Beckmann et al., 2015). We contribute to this literature by examining the predictive value of realized moments for gold futures returns across alternative forecast horizons, after controlling for well documented market-based variables and widely studied measures of investor sentiment and uncertainty (Da et al., 2015; Baker et al., 2015). More specifically, we trace out the incremental predictive value of realized volatility and skewness for gold futures returns using a recursively estimated quantile-boosting approach recently used in the literature on gold-price dynamics by Pierdzioch et al. (2016). The approach accounts for model uncertainty as well as model instability and allows the predictive value of realized moments to be analyzed across the quantiles of the conditional distribution of gold futures returns that represent different market conditions. By doing so, the analysis provides new insights to the predictability of gold futures return that can be useful in hedging and safe-haven analyses.

Research in gold price dynamics and how they relate to stock and bond market returns has recently experienced renewed interest, particularly following the 2007/08 financial crisis. Consequently, a number of papers have been published in recent years examining the diversification benefits of gold investments. Traditionally, gold has been studied as a hedge against inflation and currency depreciation (e.g., Christie-David et al., 2000; Capie et al., 2005; Worthington and Pahlavani, 2007; Blose, 2010). Following the market turmoil experienced during the credit crunch of 2008, a number of recent studies have also looked into the diversification and safehaven benefits of gold for stock and bond portfolios (e.g., Baur and Lucey, 2010; Baur and McDermott, 2010; Hood and Malik, 2013; Bredin et al., 2015). While recent studies present mixed evidence in regards to the dominance of gold as a safe haven compared to other assets (e.g., Hood and Malik, 2013; Agyei-Ampomah et al., 2014), the literature generally suggests that gold can work as a hedge and/or safe haven for stock and bond investors both in the U.S. and in other developed markets. Clearly, modeling gold returns and identifying the market variables that might have predictive value for gold-price fluctuations is of practical concern for 
hedgers and portfolio managers in the implementation of dynamic diversification and/or hedging strategies. Identification of primary determinants of gold returns can also help enlarge our understanding of volatility transmissions between gold and other market segments, which can especially be useful in cross-hedging strategies. Finally, the findings on gold return dynamics can be employed in further forecasting exercises given the recent evidence that gold prices can help forecast real exchange rates, particularly in the case of major commodity exporters (e.g., Apergis, 2014).

A significant advantage of the quantile boosting approach utilized in this study is that it follows an iterative model-building procedure in which the forecasting model is built from alternative competing predictor variables. In addition to some of the well-documented marketbased variables as well as investor sentiment and uncertainty indicators, we also examine the predictive value of higher moments measured by the realized volatility and realized skewness, which we compute using intraday return data. We are particularly interested in the predictive ability of realized volatility and realized skewness as recent research documents that higherorder moments may contain significant information regarding future returns and volatility in stock markets. While studies including Bollerslev et al. (2013) and Corsi et al. (2013) use realized volatility for forecasting stock-market returns and to develop option-valuation models, a number of studies in the asset-pricing literature underline the predictive ability of realized skewness for stock returns. Earlier studies including Barberis and Huang (2004), Brunnermeier et al. (2007), Mitton and Vorkink (2007) and Boyer et al. (2010) suggest a link between the skewness of individual securities and investors' portfolio decisions, while Bali et al. (2008) utilize skewness in Value at Risk estimations. In cross-sectional tests, Xing et al. (2010) find that portfolios sorted on a measure related to idiosyncratic skewness generate differences in returns while studies including Barberis and Huang (2008), Conrad et al. (2013) and Amaya et al. (2015) show that realized skewness has predictive value over subsequent returns. More recently, Kraussl et al. (2016) associate skewness with crash risk, enlarging the scope of risk proxies skewness may be associated with. Hence, we are primarily motivated by the evidence suggesting that it is important to account for higher moments when analyzing return dynamics, and the quantile-boosting approach employed in this study provides an appropriate framework that allows the predictive value of realized moments to be examined in the presence of other well documented market- and sentiment-based indicators.

Our findings suggest that realized moments can significantly improve the predictive value of the estimated forecasting models, even after controlling for widely studied market- and sentiment-based variables. Comparing alternative model specifications that include marketbased variables such as the nominal interest rate, term spread, exchange rates, oil and stock market returns as well as popular uncertainty and sentiment indicators, we find that a boosted model that includes realized volatility and skewness often outperforms a simple boosted AR(1) 
model as well as a boosted model that excludes the realized moments from the list of predictor variables. The predictive value of realized moments is particularly evident for intermediate forecast horizons and holds in many cases for lower quantiles, suggesting that realized moments must be taken into account in forecasting exercises that target distressed market periods in particular. This is especially important for the estimation of tail-risk measures and Value at Risk projections for periods of market stress.

We organize the remainder of the paper as follows. In Section 2, we explain the quantileboosting approach and how we evaluate the accuracy of forecasts. In Section 3, we present the data, and we explain how we compute the realized moments. In Section 4, we summarize our empirical results. In Section 5, we conclude with a discussion of practical implications of our empirical results.

\section{Methodology}

\subsection{The Quantile-Boosting Approach}

The starting point of the quantile-boosting approach (see Fenske et al., 2011; Zheng, 2012; Yuan, 2015; for a least-absolute error boosting approach, see Friedman, 2001) is the following quantile-regression period-loss function which is standard in the quantile-regression literature (on quantile regressions, see Koenker, 2005):

$$
\mathcal{L}\left(\alpha, \hat{u}_{t+1, \alpha, h}\right)=\hat{u}_{t+1, \alpha, h}\left(\alpha-\mathbf{1}\left(\hat{u}_{t+1, \alpha, h}<0\right)\right),
$$

where $\mathbf{1}(\cdot)$ denotes the indicator function, $\hat{u}_{t+1}$ is the forecast error, and the quantile parameter can assume values in the interval $\alpha \in(0,1)$. A symmetric loss function obtains for $\alpha=0.5$, while for $\alpha<0.5(\alpha>0.5)$, the loss of a negative forecast error exceeds (falls short of) the loss of a positive forecast error. Because the optimal forecast depends on the quantile parameter, the forecast error carries an $\alpha$-index. For a given $\alpha$-index, the forecast error for a given forecast horizon, $h$, is computed as $\hat{u}_{t+1, \alpha, h}=r_{t+h}-F\left(\hat{\beta}_{\alpha, h}, x_{t}\right)$, where $F\left(\beta_{\alpha, h}, x_{t}\right)=$ $\hat{\beta}_{0, \alpha, h}+\hat{\beta}_{1, \alpha, h} x_{1, t} \ldots+\hat{\beta}_{n, \alpha, h} x_{n, t}$, where $r_{t+h}$ denotes gold futures returns between period of time $t$ and $t+h$ and $\hat{\beta}_{j, \alpha, h}, j=0,1,2, \ldots, n$ denote estimates of quantile-specific regression coefficients of the predictors, $x_{t}$.

Similar to Pierdzioch et al. (2016), we use the quantile-boosting approach to estimate the regression coefficients and to decide which predictors to include in the optimal forecasting model. To this end, we select for any given $\alpha$ an optimal $F\left(\hat{\beta}_{\alpha, h}, x_{t}\right)^{*}$ by solving

$$
F\left(\hat{\beta}_{\alpha, h}, x_{t}\right)^{*}=\arg \min _{F\left(\hat{\beta}_{\alpha, h}, x_{t}\right)} E[\mathcal{L}(\alpha, h, t)]
$$


where expectations, $E$, are computed in terms of $\mathcal{L}(\alpha, h, t)=\sum_{j=0}^{t} \mathcal{L}\left(\alpha, \hat{u}_{j+1, \alpha, h}\right)$. The minimization problem given in Equation (2) is solved by means of a functional-gradient-descent boosting algorithm (Friedman, 2001; Fenske et al., 2011; for a survey of boosting algorithms, see Bühlmann and Hothorn, 2007). The algorithm is implemented by iterating over the following steps:

1. Set $m=1$ and choose a starting value. Like Fenske et al. (2011), we choose the median of the response variable as a starting value. We use demeaned data (see Bühlmann and Hothorn, 2007).

2. Compute the negative gradient vector $\hat{u}_{t+1, \alpha, h}=-\partial \mathcal{L}(\alpha, t) / \partial F\left(\hat{\beta}_{\alpha, h}^{[m-1]}, \alpha, x_{t}\right)$, where $\hat{u}_{t+1, \alpha, h}=\alpha$ if $\hat{u}_{t+1, \alpha, h} \geq 0$ and $\hat{u}_{t+1, \alpha, h}=\alpha-1$ if $\hat{u}_{t+1, \alpha, h}<0$.

3. Fit the individual elements of $x_{t}$ to the negative gradient vector. Choose the best element, $\kappa$, as the one that solves $\min L_{2}$ (see Friedman, 2001).

4. Update the vector of regression coefficients $\hat{\beta}_{\alpha, h}^{[m]}=\hat{\beta}_{\alpha, h}^{[m-1]}+v \hat{\gamma}_{\alpha, h}^{[\kappa, m]}$, where $v \in(0,1]$ denotes a step-size parameter, and $\hat{\gamma}_{\alpha, h}^{[\kappa, m]}$ contains as the only non-zero element the coefficient estimated for $\kappa$. Like Fenske et al. (2011) and others, we set $v=0.1$. Smaller values of $v$ lead to more boosting iterations.

5. Update $m$. Terminate the updating when a final iteration is reached. We determine the final iteration, $m^{*}$, as the one that minimizes the loss function. Similar to Mayr et al. (2012) and Pierdzioch et al. (2016), we run the algorithm $m_{\text {break }}$ times. If $m^{*}=\arg \min _{m} \mathcal{L}^{m}(\alpha, h, t)$ satisfies $m^{*} \leq 0.75 \times m_{\text {break }}$, we stop, where $\mathcal{L}^{m}(\alpha, h, t)$ denotes the loss in iteration $m$, given $\alpha$ and $h$. Otherwise, we set $m_{\text {break }}=m_{\text {break }}+10$, and check again if $m^{*} \leq 0.75 \times m_{\text {break }}$. We continue until we reach $m_{\max }=500$, but the algorithm typically stops much earlier.

Finally, we insert the updated predictors, $x_{t+1}$, into $F\left(\hat{\beta}_{\alpha, h}^{\left[m^{*}\right]}, x_{t+1}\right)^{*}$, and compute an out-ofsample forecast of gold futures returns. Because the optimal forecasting model may change over time we use a recursively expanding estimation window to implement the quantileboosting approach (see Pierdzioch et al., 2016).

\subsection{Forecast Evaluation}

We check the informational value of the boosted forecasts by comparing them with the forecasts from a recursively estimated boosted benchmark model, $b$. In doing so, we account for the fact that the quantile-boosting algorithm adjusts forecasts depending on the shape of the loss 
function given in Equation (1) (see Pierdzioch et al., 2015, 2016). Setting $\alpha>0.5$ implies that the loss of a positive forecast error is larger than the loss of a negative forecast error of the same absolute size, requiring an upward adjustment of forecasts relative to the case of a symmetric loss function $(\alpha=0.5)$. Similarly, a quantile parameter of $\alpha<0.5$ implies that the loss of a negative forecast error exceeds the loss of a positive forecast error, requiring a downward adjustment of forecasts relative to the symmetric benchmark case. As a result, it is important to condition any evaluation of the informational value of forecasts on the quantile parameter. We do so by extending the approach proposed by Fair and Shiller (1990) to a quantile-regression setting (Koenker and Bassett, 1978; Koenker, 2005) and estimate, separately for every combination of $\alpha$ and $h$, the following quantile-regression model (see also the analysis by Gupta et al., 2016):

$$
\gamma_{\alpha, h}^{*}=\underset{\gamma_{\alpha, h}}{\arg \min } \sum_{t} \mathcal{L}\left(\alpha, r_{t+h}-\mathbf{X}_{t+1} \gamma_{\alpha, h}\right),
$$

where the summation runs over the out-of-sample forecasting periods, $\mathbf{X}_{t+1} \gamma_{\alpha, h}=\gamma_{0, \alpha, h}+$ $\gamma_{1, \alpha, h} \hat{r}_{t+h}+\gamma_{2, \alpha, h} \hat{r}_{t+h}^{b}$, and $\mathcal{L}_{\alpha}$ is of the format of the loss function given in Equation (1). If the boosted period- $t$ forecasts, $\hat{r}_{t+h}$, have predictive value for $r_{t+h}$, and the predictive value of the period- $t$ benchmark forecasts, $\hat{r}_{t+h}^{b}$, is completely embedded in $\hat{r}_{t+h}$, then the estimate of $\gamma_{1, \alpha, h}$ should be significantly different from zero while the estimate of $\gamma_{2, \alpha, h}$ should not. If both forecasts contain non-overlapping information for $r_{t+h}$ then both coefficients, $\gamma_{1, \alpha, h}$ and $\gamma_{2, \alpha, h}$, should be significantly different from zero. If both forecasts do not have predictive value for $r_{t+h}$ then both coefficients should not be significantly different from zero. Finally, if both forecasts contain the same information for $r_{t+h}$ then the coefficients, $\gamma_{1, \alpha, h}$ and $\gamma_{2, \alpha, h}$, are not separately identified (see Fair and Shiller, 1990, page 377). Because of the overlapping structure of the data in case of multiperiod forecasts, we use bootstrap simulations to assess the significance of the coefficients.

\section{Data}

\subsection{Gold Futures Returns and Realized Moments}

Gold futures are traded in NYMEX over a $24 \mathrm{~h}$ trading day (pit and electronic). We focus on gold futures prices, rather than spot prices, due to the low transaction costs associated with futures trading, which makes the analysis more relevant for practical applications in the context of hedging and/or safe-haven analyses. Furthermore, as Shrestha (2014) notes, one can expect price discovery to take place primarily in the futures market as the futures price responds to new information faster than the spot price due to lower transaction costs and ease of short selling associated with the futures contracts. The futures price data, in continuous 
format, are obtained from www.disktrading.com. Close to expiration of a contract, the position is rolled over to the next available contract, provided that activity has increased. Daily returns are computed as the end of day (NYT) price difference (close to close). In the case of intraday returns, 1-minute prices are obtained via last-tick interpolation (if the price is not available at the 1-minute stamp, the previously available price is imputed). 1-minute returns are then computed by taking the log-differences of these prices and these intra-day returns are used to compute the realized moments. Our data set spans the period of February 2004 to February 2011 , reflecting data availability of the investor sentiment index used as one of the predictors (see Section 3.2).

We use the classical estimator of realized volatility, i.e. the sum of squared intraday returns, as per Andersen and Bollerslev (1998), realized volatility (RV) is expressed as

$$
R V_{t}=\sum_{i=1}^{M}\left(r_{t, i}\right)^{2}
$$

where $r_{t, i}$ is the intraday $M \times 1$ return vector and $i=1, \ldots, M$ is the number of intraday returns. We have $M=1,440$ minutes returns for each trading day. The realized skewness estimator is computed as

$$
R S K_{t}=\frac{\sqrt{M} \sum_{i=1}^{M}\left(r_{i, t}\right)^{3}}{R V_{t}^{3 / 2}}
$$

where $R V_{t}$ is the realized variance estimator given in Equation (4). In Section 4.3, we shall report, as a robustness check, results for alternative widely-studied estimators of realized moments.

Panel A of Figure 1 plots continuously compounded daily gold futures returns. The sample minimum of daily returns is -0.08 , the maximum is 0.09 , the standard deviation is 0.01 , and the sample mean and median are approximately zero. Based on the Jarque-Bera test statistic (not reported), we can reject normality of the sampling distribution of returns at the highest levels of significance, which provides some preliminary justification for modeling the quantiles rather than simply the mean of the conditional distribution of returns. By the same token, an analysis by means of the BDS test (Brock et al., 1996; results are available upon request) indicates, for various embedding dimensions, the presence of nonlinearity in the returns series, further strengthening the case for a quantiles-based modeling approach.

- Please include Figure 1 about here. -

Examining the plots for realized moments presented in Figure 1, we observe several notable spikes in the realized volatility of gold futures returns, particularly during 2006 and at the 
height of the Global Financial Crisis in 2008 which was a major shock to the global financial system. While it is not surprising to observe increased volatility in the gold market during the 2008 market crisis period as investors flocked to this safe haven in order to protect the value of their portfolios, the spike in volatility during 2006 can be attributed to the increased involvement of professional investors like hedge funds as gold presented an attractive investment opportunity, rather than a safe haven, outperforming the stock market during much of the 2000-2006 period.

The plot for realized skewness presented in Panel C, on the other hand, is characterized by notable positive spikes in realized skewness during the pre-crisis period, reflecting the sustained rise in gold prices and possibly some exuberance during this period as Figuerola-Ferretti and McCrorie (2016) note. Interestingly, the outbreaks in realized skewness do not exactly match those observed for realized volatility in Panel B, which suggests some degree of separation in the informational content of these two moments for gold return dynamics. In the aftermath of the global financial crisis period, however, negative outbreaks in realized skewness become more evident in the general pattern, reflecting several important developments in financial markets including news from China in April of an increase in its gold reserves, the IMF's sale of gold in August in the context of rising gold prices, and later in 2011, news of stability in the Eurozone thanks to the approval of austerity cuts in Greece. Overall, the difference in the patterns observed in the plots for realized volatility and skewness underline the difference in the informational content these moments might have and further motivates the analysis regarding their predictive value for gold futures returns.

\subsection{Other Predictor Variables}

In addition to realized volatility and realized skewness, we consider several market- and sentiment-based predictors in the construction of the forecasting models. The list of the predictors comprises determinants of gold returns widely studied in earlier research, but also includes measures of investor sentiment and uncertainty. Particularly motivated by Kraussl et al. (2016) that skewness may be associated with crash risk, we control for rival measures of investor sentiment and market uncertainty in our iterative model-building procedure in order to assess the incremental predictive value of realized moments for gold futures returns. This approach renders it possible to quantify the incremental predictive value of realized moments in the presence of other predictor variables that may contain valuable information regarding the state of the market, in particular distressed market periods. Below, we provide a brief description of the additional predictor variables that we utilize in our analysis:

- Investor sentiment. In a recent study, Da et al. (2015) use daily data on Internet search volume from millions of households to construct an index of market-level sentiment. By 
aggregating the volume of queries related to household concerns (for example, "recession", "unemployment", and "bankruptcy"), they construct a Financial and Economic Attitudes Revealed by Search (FEARS) index as a new measure of investor sentiment. Da et al. (2015) show that the FEARS index has predictive power over short-term stock market reversals as well as temporary increases in volatility. Their data covers the daily period of 1st July, 2004 to December 31th, 2011, and to the best of our knowledge is the only available investor-sentiment index at daily frequency. Naturally, our analysis is restricted to this sample period.

- Nominal interest rate. A number of studies including Hammoudeh and Yuan (2008), Bhar and Hammoudeh (2011), and Pierdzioch et al. (2014) have suggested the nominal interest rate as a determinant of gold returns and volatility. For this reason, we use the 3-month T-bill rate as the short-term nominal interest rate and stochastically detrend the short-term interest rate by its one-month backward looking average (see also, for example, Rapach et al. 2005).

- Term spread. The term spread, defined as the difference between the yield on 10-year government bonds and the 3-month T-bill rate, is also considered as a driver of gold price fluctuations (e.g. Pierdzioch et al., 2014; Bialkowski et al., 2015) as it may contain information regarding the market's inflationary expectations as well as future economic growth prospects.

- Exchange rates. The role of exchange rate movements for gold returns has been examined in a number of studies including Pukthuanthong and Roll (2011), Reboredo (2013b), and Reboredo and Rivera-Castro (2014). We use in our empirical analysis the continuously compounded returns for the dollar/pound and the yen/dollar exchange rates.

- Stock market variables. As mentioned earlier, stock market returns play a key role in studies that deal with hedging and safe haven benefits of gold investments (Baur and Lucey, 2010; Baur and McDermott, 2011; Beckmann et al., 2015). We use continuously compounded returns on the S\&P 500 index (closing prices) and the CBOE S\&P 100 volatility index (VXO), where the latter measures the expected intensity of market fluctuations.

- Oil price. Several recent studies including Zhang and Wei (2010), Beckmann and Czudaj (2013), Malliaris and Malliaris (2013), and Ewing and Malik (2013) study the link between the price of gold and the price of oil. We use continuously compounded oil-price returns (West Texas Intermediate) to represent oil price fluctuations.

- Economic uncertainty. We use the daily equity market uncertainty (EMU) and economic policy uncertainty (EPU) indices for the U.S. economy as developed by Baker 
et al. (2015) to measure economic uncertainty. The daily news-based indexes use newspaper archives from Access World New's NewsBank service. The primary measure for this index equals the number of articles that contain at least one term from each of three sets of terms (economic or economy, uncertain or uncertainty, and legislation, regulation, Congress, Federal Reserve, or White House). Using the same news source, the EMU index searches for articles containing the terms uncertainty or uncertain, economic or economy, and one or more of the following terms: equity market, equity price, stock market, or stock price. Data on these two indices come from the website: http://www.policyuncertainty.com.

- Contemporaneous daily gold futures returns. Including gold futures returns implies that the quantile-boosting approach can select a simple AR(1) forecasting model in case the other predictor variables do not have predictive value for subsequent gold futures returns.

\section{Empirical Results}

\subsection{Structure of the Forecasting Models}

For computing our baseline results, we use $75 \%$ of the data (1,222 observations; the initialization period ends in July 2009; as a robustness check, we shall present results for an extended out-of-sample period in Section 4.4) to initialize the quantile-boosting approach, and the remaining data for out-of-sample forecasting. In total, we have 408 out-of-sample forecasts for every forecast horizon and quantile.

- Please include Figure 2 about here. -

Figure 2 summarizes the number of iterations it takes to find the minimum of the empirical loss function and the number of predictors selected by the quantile-boosting approach (results averaged across out-of-sample forecasting periods). A general pattern that emerges is that it takes more iterations to find the minimum of the loss function for the lower and upper quantiles than for quantiles closer to the median. For the longer forecasting horizon (ten-days-ahead), this pattern becomes asymmetric insofar as the lower quantiles require more iterations than the upper quantiles.

Another pattern that emerges from Figure 2 is that it takes more iterations to find the minimum of the loss function for the longer forecast horizons. In line with this pattern, the quantile-boosting approach selects more predictors for the longer forecast horizons. For oneday-ahead returns, the quantile-boosting approach mainly selects predictors for the extreme 
lower and upper quantiles. The reason for why the quantile-boosting approach runs only few iterations and, at the same time, selects no predictors is that, in the case of one-day-ahead returns, the empirical loss function is very irregular for some quantiles, and so we do not push the interpretation of the results further for these quantiles. For five-days-ahead returns, the number of selected predictors increases, but we still observe the pattern that the boosted forecasting models tend to include more predictors as we move farther away from the median. For the ten-days-ahead returns, this pattern becomes less pronounced and asymmetric.

- Please include Figure 3 about here. -

Figure 3 shows how often the boosted forecasting models feature the realized moments (results averaged across out-of-sample forecasting periods). For one-day-ahead returns, realized volatility mainly enters the boosted forecasting models for several upper and the two lower quantiles. Realized skewness, in turn, enters the boosted forecasting models only at the two lowest quantiles, underlining the predictive value of skewness particularly for distressed market states and tail risk measures. The predictive value of skewness, particularly during periods of market stress represented by extreme lower quantiles, has significant implications for safe haven analyses and portfolio diversification strategies involving positions in gold as a number of studies have documented the importance of fat tails in asset-allocation decisions (e.g., Xiong and Idzorek, 2011).

For one-day-ahead returns, we observe, in terms of the frequency of inclusion in the boosted forecasting models, that realized volatility is generally more dominant than realized skewness, although its importance as a predictor varies based on the returns quantile considered, further supporting the importance of a quantile-based model building approach in forecasting exercises. For five-day-ahead returns, realized volatility still enters very often the boosted forecasting models at the upper quantiles, and it gains in importance for the quantiles below the median. Similarly, the importance of realized skewness substantially increases to up to approximately $80 \%$ for several of the quantiles below the median, and it is included very often in the boosted forecasting models for the extreme upper quantile.

In the case of ten-day-ahead returns, both realized moments still enter the boosted forecasting models often for $\alpha=0.85$ and $\alpha=0.9$. In addition, for quantiles below $\alpha \leq 0.55$, the boosted forecasting models always feature realized volatility. The importance of realized skewness increases for the quantiles in the range $0.55 \leq \alpha \leq 0.7$ and remains strong, and in some cases strengthens even further relative to the results for five-days-ahead returns, for quantiles below $\alpha=0.4$. Overall, the findings clearly point to the predictive value contained in realized moments with quantile- and forecasting-horizon-based patterns that should be taken into account in forecasting exercises. 


\section{$4.2 \quad$ Fair-Shiller Regressions}

A natural question is whether the quantile-boosting approach outperforms a simple boosted AR(1) model, which the quantile-boosting model can select as a special case because we include lagged gold futures returns in our list of potential predictors. Table 1 summarizes the results of Fair-Shiller regresssions where we compare a boosted forecasting model that includes the realized moments in the list of potential predictors with a recursively estimated boosted AR(1) model. For one-day-ahead returns, we have NA entries because the quantile-boosting approach selects no predictors for some quantiles. For those quantiles for which results are available, the Fair-Shiller regressions do not yield significant results for both the quantile-boosting approach and the boosted AR(1) model (with one exception). In contrast, for five-days-ahead returns, the quantile-boosting approach dominates the boosted AR(1) model for several of the lower ( $\alpha \leq 0.3$, except for the lowest quantile) and some upper quantiles $(\alpha \geq 0.75$, except for the largest quantile). For ten-days-ahead returns the dominance of the quantile-boosting approach becomes stronger for the lower quantiles, while results for the quantiles above the median are not significant for the quantile-boosting approach.

- Please include Tables $1-2$ about here. -

Table 2 summarizes the results of Fair-Shiller regressions that compare a boosted model that excludes the realized moments from the list of predictors and a boosted $\operatorname{AR}(1)$ model. We observe that excluding the realized moments from the list of predictors worsens the predictive value of five-days-ahead forecasts relative to the boosted $\mathrm{AR}(1)$ benchmark model for quantiles below the median. For few quantiles (mainly $\alpha=0.75$ and $\alpha=0.8$ ) above the median, we still observe significant results. Similarly, deleting the realized moments from the list of potential predictor variables deteriorates the relative performance of the boosted model for the quantiles below the median in the case of ten-day-ahead returns.

- Please include Table 3 about here. -

Table 3 summarizes the results of a direct horserace between the forecasts generated using a boosted model that includes the realized moments in the list of potential predictors with the forecasts from a boosted model that excludes the realized moments. While the models do not differ systematically in terms of their predictive value for one-day-ahead returns, the model that accounts for the realized moments clearly dominates at a five-day-ahead forecast horizon for the lower quantiles $(\alpha \leq 0.2)$. For a ten-day-ahead forecast horizon, this dominance strengthens and extends to quantiles below $\alpha \leq 0.3$. 
Overall, the evidence suggests that realized moments contain significant predictive value in forecasting returns, even in the presence of other well documented market- and sentimentbased predictors. In particular, the finding that realized skewness serves as a major driver of forecasting performance for gold returns over the subsequent week is consistent with previous research on stock returns that idisonsyncratic skewness has predictive power over future returns (e.g., Xing et al, 2010; Conrad et al., 2013) and research on commodities that skewness can significantly improve the quality of Value at Risk estimates (e.g. Cheng and Hung, 2016). From a behavioral perspective, the observed predictive value of skewness even in the presence of other market variables can be related to what Barberis and Huang (2008) suggest as investors' preference over highly skewed payoffs, which may be particularly relevant for gold. Indeed, the finding that realized skewness is important particularly at extreme quantiles, and at quantiles below the median, can be due to investors' tradeoff between the benefits of diversification and skewness, as Brunnermeier et al. (2007) and Barberis and Huang (2008) suggest, which can particularly be prevalent during distressed market conditions, leading to the observed predictive power skewness possesses during such market states. Another possible explanation for the predictive value of skewness is that investors tend to hold undiversified portfolios, as noted by Mitton and Vorkink (2007), and thus tend to overinvest in positively skewed assets during periods of market stress, which may particularly be the case for safe haven assets such as gold. Nevertheless, whatever the underlying economic rationale might be, our findings clearly point to the significant predictive value of realized moments during distressed market periods, even in the presence of other well cited market- and sentimentbased predictors for gold returns. To that end, our findings provide novel insight to the role of market sentiment in the market for commodities in that realized moments may contain valuable information that relates to what Gao and Suss (2015) term as sentiment exposure in commodity futures returns.

\subsection{Alternative Realized Moments}

We next study the robustness of our results to a change in how we measure realized moments. We consider two alternative measures of realized moments. The first alternative measure we use, which is robust to market-microstructure noise and asynchronous trading, is the realizedkernel estimator of Barndorf-Nielsen et al (2007). We use the realized-kernel estimator to compute the realized volatility as follows:

$$
R V_{t}=\sum_{h=-H}^{H} k\left(\frac{h}{H+1}\right) \gamma_{h}, \quad \gamma_{h}=\sum_{j=|h|+1}^{M} r_{t, j} r_{t, j-|h|},
$$

where $k(\cdot)$ is a kernel function and $H$ is the bandwidth parameter, $r_{t, j}$ is the $j$-th high frequency return with $j=1, \ldots, M$ for day $t$. We use a flat-top kernel function with $H=1$. 
A second alternative measure is the two-times-scales estimator proposed by Zhang et al. (2005). This estimator uses a slow time scale, $K$, to estimate the realized volatility (via subsampling) and a fast time scale, $J$, to correct for the presence of market micro-structure noise. We have

$$
R V_{t}=\left(1-\frac{\bar{M}_{K}}{\bar{M}_{J}}\right)^{-1}\left([X, X]_{t}^{(K)}-\frac{\bar{M}_{K}}{\bar{M}_{J}}[X, X]_{t}^{(J)}\right),
$$

where $\bar{M}_{K}=(M-K+1) / K, \bar{M}_{J}=(M-J+1) / J$ and

$$
[X, X]_{t}^{(K)}=\frac{1}{K} \sum_{i=1}^{M-K+1}\left(X_{t, i+K}-X_{t, i}\right)^{2}
$$

with $X_{t, i}$ the $i$-th intraday price for day $t$ and $M$ the number of intra-day prices. For this estimator we use 10-minute returns as slow scale and 1-minute returns as fast scale. For both estimators, we insert $R V_{t}$ into Equation (5) to compute realzed skewness.

- Please include Tables 4 and 5 about here. -

The results of Fair-Shiller regressions (for five-days-ahead and ten-days-ahead returns) summarized in Tables 4 and 5 corroborate the results based on the classical measures of realized moments. The boosted model that features the realized moments in the list of potential predictors tends to fare better than a boosted $\mathrm{AR}(1)$ model for some of the quantiles below the median, whereas the boosted model that excludes the realized moments from the list of potential predictors does not outperform an AR(1) benchmark model. A horserace between the boosted models that include/exclude the realized moments as predictors of gold future returns shows that, for quantiles in the approximate interval $\alpha \leq 0.2$, the coefficient of the forecasts that account for the predictive value of the realized moments for five-days-ahead returns is significant, while the coefficient of the benchmark model that excludes the realized moments is not significant. For ten-days-ahead returns, this interval widens to approximately $\alpha \leq 0.4$. For quantiles $0.25 \leq \alpha \leq 0.35$ both coefficients are significant.

\subsection{Extended Forecasting Period}

Having presented evidence on the predictive ability of realized moments for the selected baseline sample period discussed in Section 4.1, we next analyze an extended out-of-sample forecasting period for a robustness check. We use this additional analysis also to shed light on the time-variation in the predictive value of the realized moments. Specifically, we reserve $50 \%$ of the data for out-of-sample forecasting (the initialization period ends in November 2007) such that the out-of-sample forecasting period comprises the onset of the financial crisis of 2008/2009. We focus on the case of five-day-ahead and ten-day-ahead returns. 
- Please include Figure 4 about here. -

Figure 4 plots the results for Fair-Shiller regressions for the case of five-days-ahead returns. We estimate the Fair-Shiller regressions on a rolling-estimation window of length 250 days. Thus, we start with the first 250 forecasts (that is, approximately one year of trading days) computed for the extended out-of-sample forecasting period and then move the rolling-estimation window forward in time on a daily basis. Every dot represents, for a given quantile, a coefficient significantly different from zero at the $5 \%$ significance level.

Comparing the results for a boosted model that includes the realized moments in the list of potential predictor variables with a boosted AR(1) model, we observe that the coefficient of the former model is significant more often than that of the boosted AR(1) model, especially at the lower quantiles. In contrast, the coefficient of the boosted $\mathrm{AR}(1)$ model is significant only occasionally and the significant coefficients are concentrated at few quantiles. At the beginning of the out-of-sample forecasting period, the coefficient of the boosted model that includes the realized moments is significant mainly for the largest quantile. As we move the estimation window forward in time, the coefficient of the boosted model loses its significance for most quantiles above the median. The coefficient, however, becomes significant for quantiles below approximately 0.3 . This finding further supports our earlier observation that realized moments possess significant predictive value particularly for distressed market states.

When we exclude the realized moments from the list of potential predictor variables, we observe a significant coefficient for the largest quantile, but only in the first half of the outof-sample period. The coefficient is significant also for a few of the other quantiles during some periods of time. However, the coefficient completely loses its significance in the second half of the out-of-sample period, and especially for all quantiles below the median. It, thus, is not surprising that the Fair-Shiller regressions show that, in the second half of the out-ofsample period, forecasts computed upon including the realized moments in the list of potential predictor variables have a better predictive value for the lower quantiles than forecasts that neglect the informational content of realized moments.

- Please include Figure 5 about here. -

In the case of ten-days-ahead forecasts, the predictive value of the realized moments is mainly concentrated at the two upper quantiles in the first half of the out-of-sample period. At the end of the out-of-sample period again the predictive value shifts to the lower quantiles. The boosted AR(1) model, in contrast, yields a significant coefficient only occasionally and mainly for the lower quantiles. When we exclude the realized moments from the list of predictor variables, we still obtain forecasts that yield a significant coefficient for the two upper quantiles 
at the beginning of the out-of-sample period, but the significance of the coefficient becomes fragile and more scattered across the quantiles as we move the rolling window forward in time. In particular, we do not observe a systematically significant coefficient for the lower quantiles in the second half of the out-of-sample period, which is in stark contrast to the results for a boosted model that inlcudes the realized moments in the list of potential predictors. Finally, when we directly compare the boosted models that include/exclude the realized moments, the significance of the coefficients for the two upper quantiles is largely lost, indicating that the information embedded in the forecasts implied by both models overlap to a substantial extent. In the second half of the out-of-sample period, the model that includes the realized moments in the list of potential predictor variables yields much more often significant results, where the significance of the coefficient is also substantially more stable across time, for quantiles smaller than approximately 0.4 than the model that never uses the information embedded in the realized moments to forecast gold futures returns.

\section{Concluding Remarks}

We have used a recursively estimated quantile-boosting approach to show that realized volatility and realized skewness have incremental predictive value for gold futures returns over and above several well-documented market-based variables as well as investor sentiment and uncertainty indicators. A significant advantage of the recursively estimated quantile-boosting approach is that it accounts for model uncertainty and model instability, and that it allows the predictive value of realized moments to be traced out across the quantiles of the conditional distribution of gold futures returns. This is particularly important for regime-based asset-allocation decisions as well as the estimation of tail-risk measures that focus on periods of market stress.

We find that realized moments often significantly improve the predictive value of the estimated forecasting models, even after controlling for widely-studied market-based variables including the nominal interest rate, term spread, exchange rates, oil and stock market returns as well as popular uncertainty and sentiment indicators. Based upon a comparison of a boosted model that includes realized volatility and skewness with alternative boosted models based on an $\mathrm{AR}(1)$ specification and a boosted model that includes only the market- and sentiment-based predictor variables, we show that realized moments can add significant predictive value for intermediate forecast horizons and also for extreme and particularly for lower quantiles of the conditional distribution of gold futures returns. This finding suggests that realized moments must be taken into account in forecasting exercises that target distressed market periods. By the same token, the findings may also serve as a guideline in regime-based asset-allocation 
strategies in which gold is utilized as a hedge (or safe haven) in order to protect portfolio value during distressed market conditions.

While the predictive value of realized moments for gold futures returns may reflect investors' preference over highly skewed payoffs or the tradeoff between diversification and skewness, particularly during periods of market stress, it is also possible that realized skewness serves as a proxy for crash risk as recently noted by Kraussl et al. (2016). To that end, it is not unexpected to find realized skewness often included in the forecasting model selected by the quantile-boosting approach. One avenue for future research, therefore, is to examine whether the realized moments for gold futures returns contain information regarding future crash risk in financial markets. 


\section{References}

Agyei-Ampomah, S., Gounopoulos D., Mazouz, K., 2014. Does gold offer a better protection against losses in sovereign debt bonds than other metals? Journal of Banking and Finance 40, 507-521.

Amaya, D., Christoffersen, P., Jacobs, K. and Vasquez, A. 2015. Does realized skewness predict the cross-section of equity returns? Journal of Financial Economics 118, 135-167.

Andersen T.G. , Bollerslev T. 1998. Answering the skeptics: Yes, standard volatility models do provide accurate forecasts. International Economic Review 39, 885-905.

Apergis, N. 2014. Can gold prices forecast the Australian dollar movements? International Review of Economics and Finance 29, 75-82.

Baker. S G. and Kramer, B. S. 2007. Peirce, Youden, and receiver operation characteristic curves. The American Statistician 61, 343-346.

Bali, T., Tang, Y., Mo, H., 2008. The role of autoregressive conditional skewness and kurtosis in the estimation of conditional VaR. Journal of Banking and Finance 32, 269-282.

Barberis, N., Huang, M., 2008. Stocks as lotteries: the implications of probability weighting for security prices. American Economic Review 98, 2066-2100.

Barndorf-Nielsen, O., Hansen, P., Lunde, A., and Shephard, N., 2008, Designing realized kernels in to measure the ex-post variation of equity prices in the presence of noise. Econometrica, 76(6), 1481-1536.

Baur, D.G., McDermott, T.K., 2010. Is gold a safe haven? International evidence. Journal of Banking and Finance 34, 1886-1898.

Baur, D.G., and Lucey, B.M., 2010. Is gold a hedge or a safe haven? An analysis of stocks, bonds and gold. Financial Review 45, 217-229.

Bhar, R., and Hammoudeh, S., 2011. Commodities and financial variables: Analyzing relationships in a changing regime environment. International Review of Economics and Finance 20, 469-484.

Beckmann, J., and Czudaj, R. 2013. Oil and gold price dynamics in a multivariate cointegration framework. International Economics and Economic Policy 10, 453-468.

Beckmann, J., Berger ,T., and Czudaj, R. 2015. Does gold act as a hedge or a safe haven for stocks? A smooth transition approach. Economic Modelling 48, 16-24. 
Bialkowski, J., Bohl, M.T., Stephan, P.M., and Wisniewski, T.P., 2015. The gold price in times of crisis. International Review of Financial Analysis 41, 329-339.

Blose, L.E., 2010. Gold price, cost of carry, and expected inflation. Journal of Economics and Business 62, 35-47.

Bollerslev, T., Osterrieder, D., Sizova, N., Tauchen, G., 2013. Risk and return: long run relations, fractional cointegration and return predictability. Journal of Financial Economics 108, 409-424.

Boyer, B., Mitton, T., Vorkink, K., 2010. Expected idiosyncratic skewness. Review of Financial Studies 23, 169-202.

Bredin, D., Conlon, T., Poti, V. 2015. Does gold glitter in the long-run? Gold as a hedge and safe haven across time and investment horizon. International Review of Financial Analysis 41, 320-328.

Brock, W. A., Scheinkman, J. A., Dechert, W. D., and LeBaron, B., 1996. A test for independence based on the correlation dimension. Econometric Reviews 15, 197-235.

Brunnermeier, M., Gollier, C., Parker,J ., 2007. Optimal beliefs, asset prices and the preference for skewed returns. American Economic Review 97,159-165.

Bühlmann, P., and Hothorn, T., 2007. Boosting algorithms: Regularization, prediction and model fitting. Statistical Science 22, 477-505.

Capie, F., Mills, T. C., Wood, G., 2005. Gold as a hedge against the dollar. Journal of International Financial Markets Institutions and Money, 15, 343-352.

Cheng, W.-H., Hung, J.-C., 2011. Skewness and leptokurtosis in GARCH-typed VaR estimation of petroleum and metal asset returns. Journal of Empirical Finance, 18 (1), $160-173$.

Christie-David R, Chaudhry M, Koch T.W. 2000. Do macroeconomics news releases affect gold and silver prices. Journal of Economics and Business 52, 405`421.

Ciner, C., Gurdgiev, C. and Lucey, B. M., 2013. Hedges and safe havens: An examination of stocks, bonds, gold, oil and exchange rates. International Review of Financial Analysis 29, 202-211.

Conrad, J. Dittmar, R. F., and Ghysels, E., 2013. Ex ante skewness and expected stock returns. Journal of Finance LXVIII, 85-124. 
Corsi, F., Fusari, N., LaVecchia, D., 2013. Realizing smiles: options pricing with realized volatility. Journal of Financial Economics 107, 284-304.

Da, Z., Engelberg, J., Gao, P. 2015. The Sum of All FEARS Investor Sentiment and Asset Prices Rev. Financ. Stud. 28 (1), 1-32.

Ewing, B. T., and Malik, F., 2013. Volatility transmission between gold and oil futures under structural breaks. International Review of Economics and Finance 25, 113-139.

Fair, R.C., and Shiller, R.J., 1990. Comparing information in forecasts from econometric models. American Economic Review 80, 375-389.

Fenske, N., Kneib, T., and Hothorn, T., 2011. Identifying risk factors for severe childhood malnutrition by boosting additive quantile regression. Journal of the American Statistical Association 106, 494-510.

Figuerola-Ferretti, I., McCrorie, R., 2016. The shine of precious metals around the global financial crisis. Journal of Empirical Finance, forthcoming.

Friedman, J., 2001. Greedy function approximation: A gradient boosting machine. Annals of Statistics 29, 1189-1232.

Gupta, R., Majumdar, A., Pierdzioch, C., and Wohar, M., 2016. Do terror attacks predict gold returns? Evidence from a quantile-predictive-regression approach. Working Paper 2016-26, University of Pretoria, Department of Economics.

Hammoudeh, S., and Yuan, Y., 2008. Metal volatility in presence of oil and interest rate shocks. Energy Economics 30, 606-620.

Hillier, D., Draper, P., and Faff, R. (2006). Do precious metals shine? An investment perspective. Financial Analysts Journal 62, 98-106.

Hood M., Malik F., 2013. Is gold the best hedge and a safe haven under changing stock market volatility? Review of Financial Economics 22, 47-52.

Koenker, R., and Basset, G., 1978. Regression quantiles. Econometrica 46, 33-50.

Koenker, R., 2005. Quantile Regression. Cambridge University Press, Cambridge.

Koenker, R., 2013. quantreg: Quantile Regression. R Package Version 5.05. http://CRAN . R-project.org/package1/4quantreg.

Kraussl, R., Lehnert, T., and Senulyte, S. 2016. Euro crash risk. Journal of Empirical Finance, forthcoming. 
Malliaris, A.G., and Malliaris, M. (2013). Are oil, gold and the euro inter-related? Time series and neural network analysis. Review of Quantitative Finance and Accounting 40, $1-14$.

Mayr, A., Hofner, B., and Schmid, M., 2012. The importance of knowing when to stop. A sequential stopping rule for component-wise gradient boosting. Methods of Information in Medicine 51, 178-186.

Mitton, T., Vorkink, K., 2007. Equilibrium under diversification and the preference for skewness. Review of Financial Studies 20, 1255-1288.

Pierdzioch, C., Risse, M., and Rohloff, S., 2014. On the efficiency of the gold market: results of a real-time forecasting approach. International Review of Financial Analysis $32,95-108$.

Pierdzioch, C., Risse, M., and Rohloff, S. 2015. A real-time quantile-regression approach to forecasting gold-price fluctuations under asymmetric loss. Resources Policy 45, 299-306.

Pierdzioch, C., Risse, M., and Rohloff, S. 2016. A quantile-boosting approach to forecasting gold returns. North American Journal of Economics and Finance 35, 38-55.

Pukthuanthong, K., and Roll, R., 2011. Gold and the dollar (and the Euro, Pound, and Yen). Journal of Banking and Finance 35, 2070-2083.

Rapach, D.E., Wohar, M.E., and Rangvid, J., 2005. Macro variables and international stock return predictability, International Journal of Forecasting 21, 137-166.

R Core Team, 2014. R: A language and environment for statistical computing, R Foundation for Statistical Computing, Vienna, Austria. URL http://www.R-project.org/.

Reboredo, J.C., 2013a. Is gold a hedge or safe haven against oil price movements? Resources Policy 38, 130-137.

Reboredo, J.C., 2013b. Is gold a safe haven or a hedge for the US dollar? Implications for risk management. Journal of Banking and Finance 37, 2665-2676.

Reboredo, M.A., and Rivera-Castro, A., 2014. Can gold hedge and preserve value when the US dollar depreciates? Economic Modelling 39: 168-173.

Roache, S. K., and Rossi, M. (2009). The effects of economic news on commodity prices: Is gold just another commodity? IMF Working Paper (WP/09/140).

Shrestha, K. 2014. Price discovery in energy markets. Energy Economics 45, 229-233. 
Süss, S., Gao, L., 2015. Market sentiment in commodity futures returns. Journal of Empirical Finance 33, 84-103.

Worthington A.C., Pahlavani M., 2007. Gold investment as an inflationary hedge: cointegration evidence with allowance for endogenous structural breaks. Applied Financial Economics Letters 3, 259-262.

Xing, Yuhang, Xiaoyan Zhang, and Rui Zhao, 2010, What does the individual option volatility smirk tell us about future equity returns?, Journal of Financial and Quantitative Analysis 45, 641-662.

Xiong, J. X., Idzorek, T. M., 2011. The impact of skewness and fat tails on the asset allocation decision. Financial Analysts Journal 67, 23-35.

Yuan, S., 2015. Random gradient boosting for predicting conditional quantiles. Journal of Statistical Computation and Simulation, 85, 3716-3726.

Zhang, L., Mykland, P., and Sahalia, Y. A., 2005, A tale of two time scales: Determining integrated volatility with noisy high-frequency data. Journal of the American Statistical Association, 100(472), 1394-1411.

Zhang, Y.-J., and Wei, Y.-M., 2010. The crude oil market and the gold market: evidence for cointegration, causality and price discovery. Resources Policy 35, 168-177.

Zheng, S., 2012. QBoost: Predicting quantiles with boosting for regression and binary classification. Expert Systems with Applications 39, 1687-1697. 


\section{Tables and Figures}

Figure 1: Gold Futures Returns and Realized Moments

Panel A: Returns

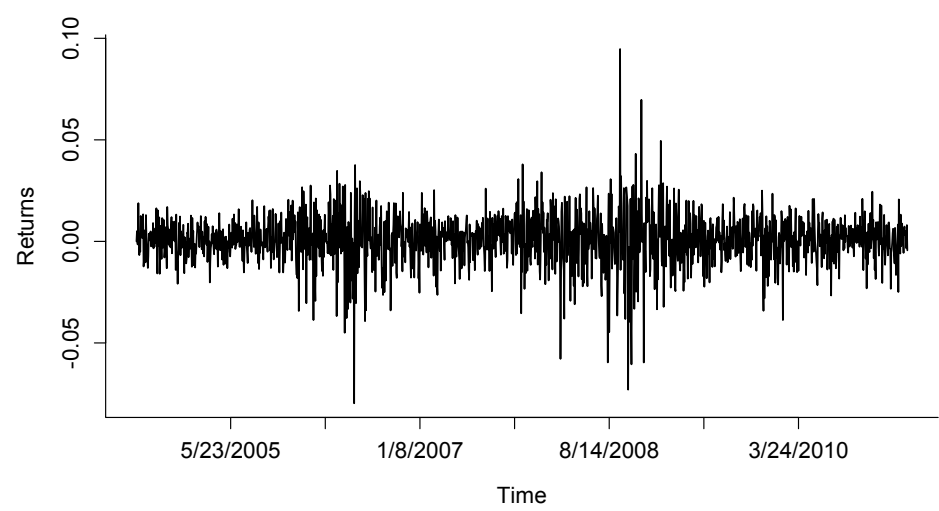

Panel B: Realized Volatility

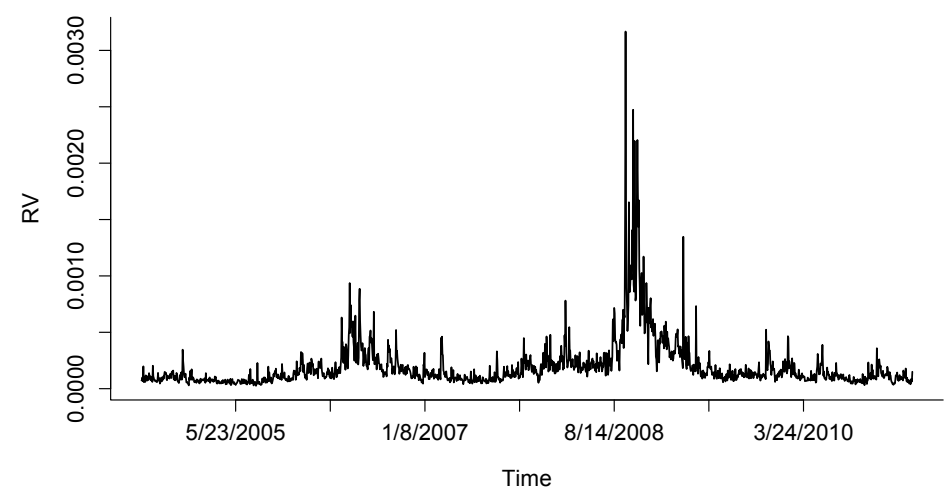

Panel C: Realized Skewness

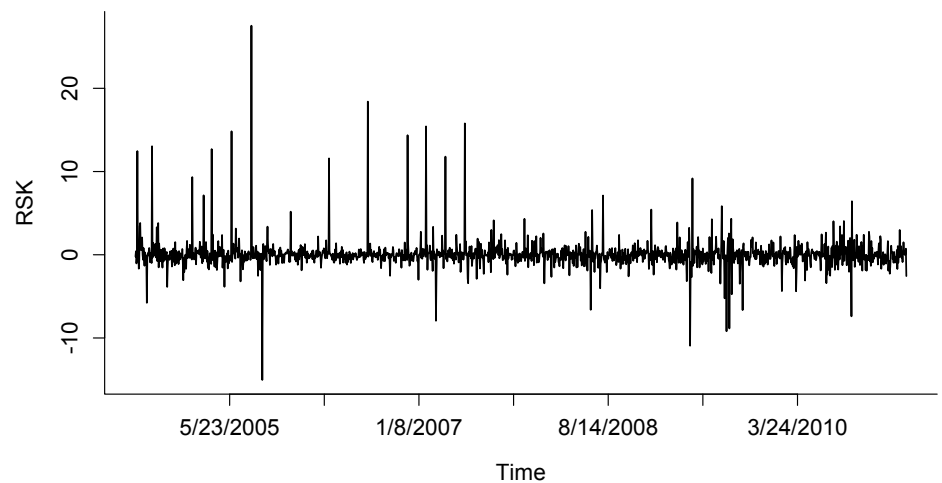

Note: This figure and all other empirical results reported in this research were computed using the free $\mathrm{R}$ programming environment (R Development Core Team 2014). 
Figure 2: Results for Alternative Forecast Horizons
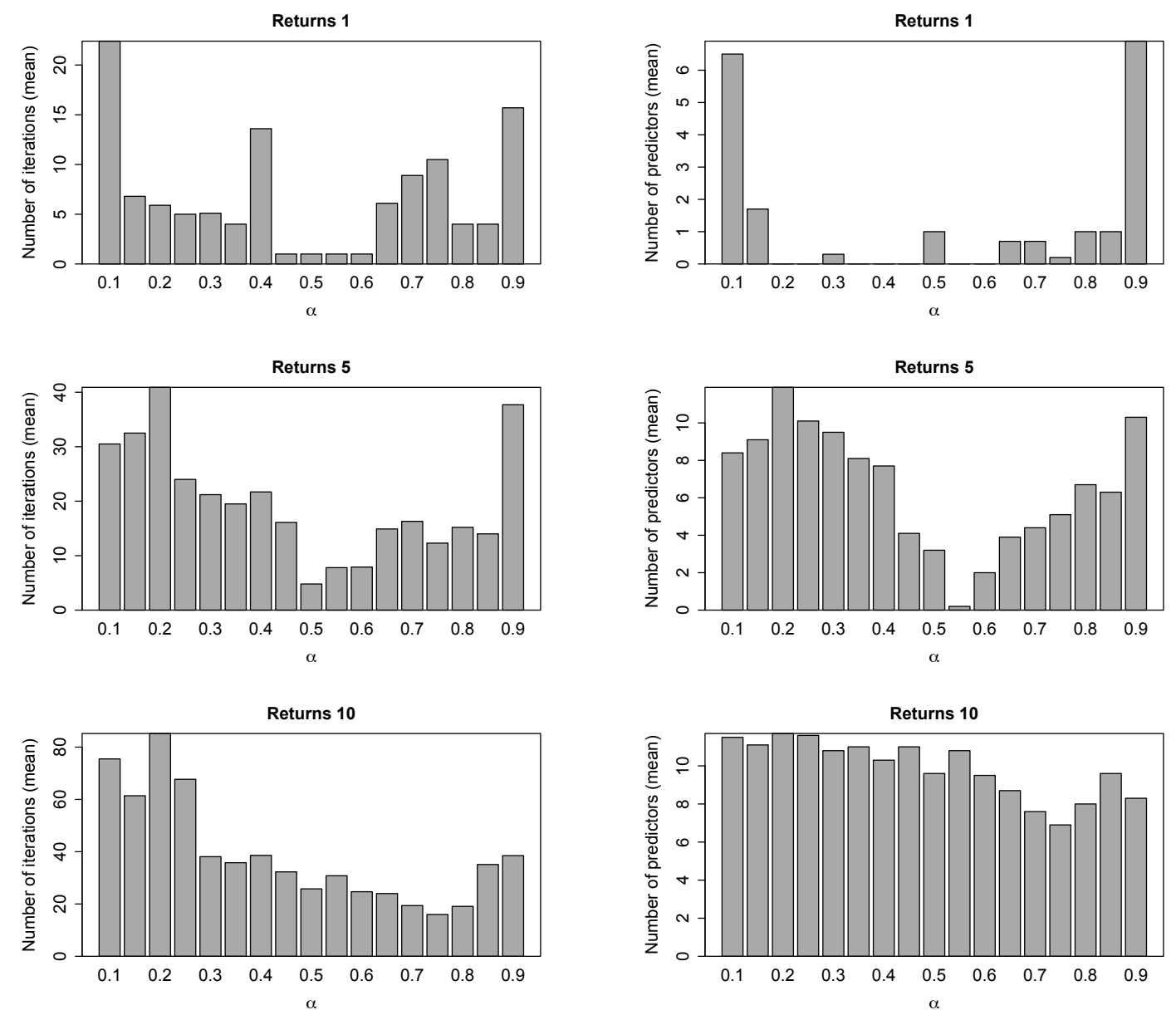

Note: Results (starting in the top row) for one-day-ahead, five-days-ahead, and ten-days-ahead returns. Results are averaged across the 408 out-of-sample periods being studied. 
Figure 3: Inclusion of Realized Moments Across Quantiles in the Forecasting Model
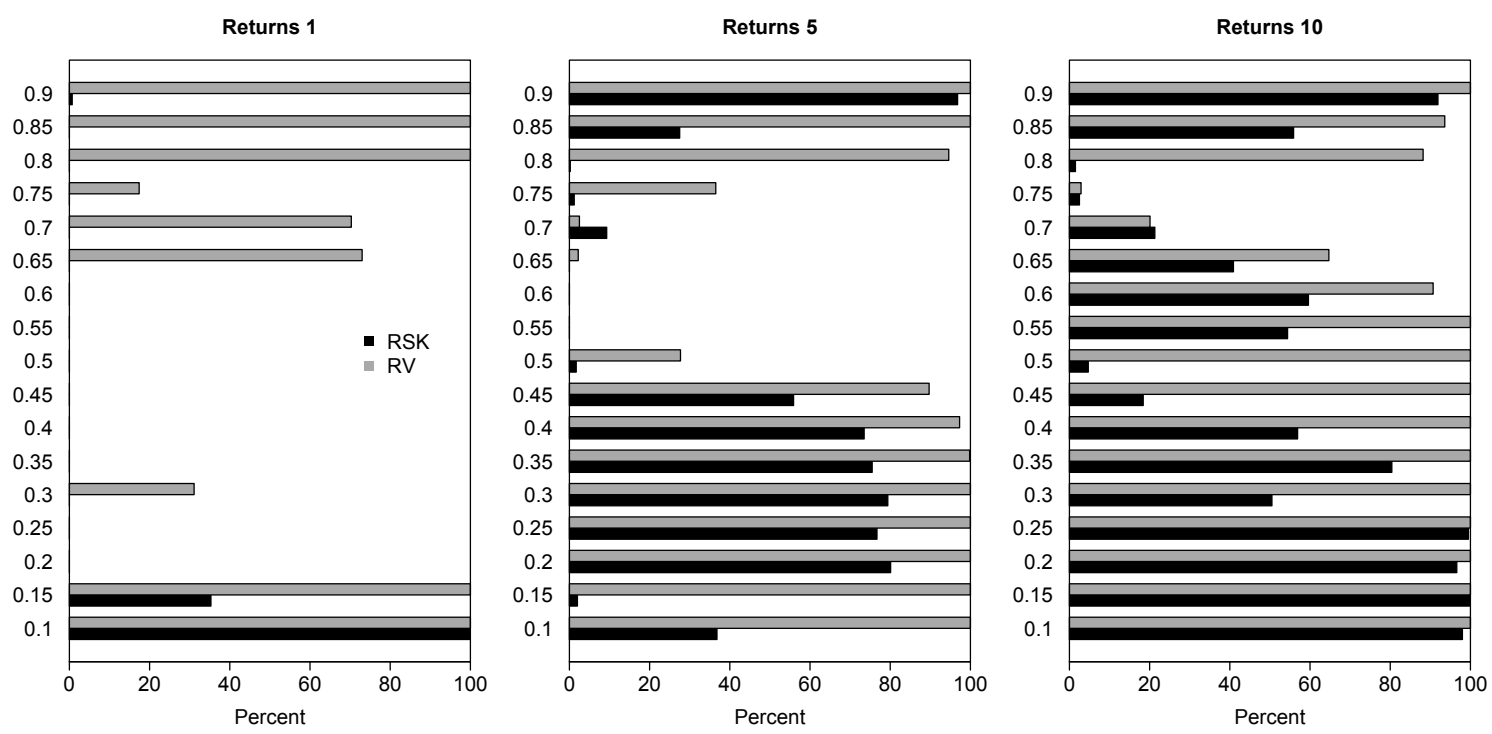

Note: Results (from left to right) for one-day-ahead, five-days-ahead, and ten-days-ahead returns. RV = realized volatility. RSK $=$ realized skewness. Results are based on the 408 out-of-sample periods being studied. 
Figure 4: Rolling Fair-Shiller Regressionens (Five-Days-Ahead Returns)
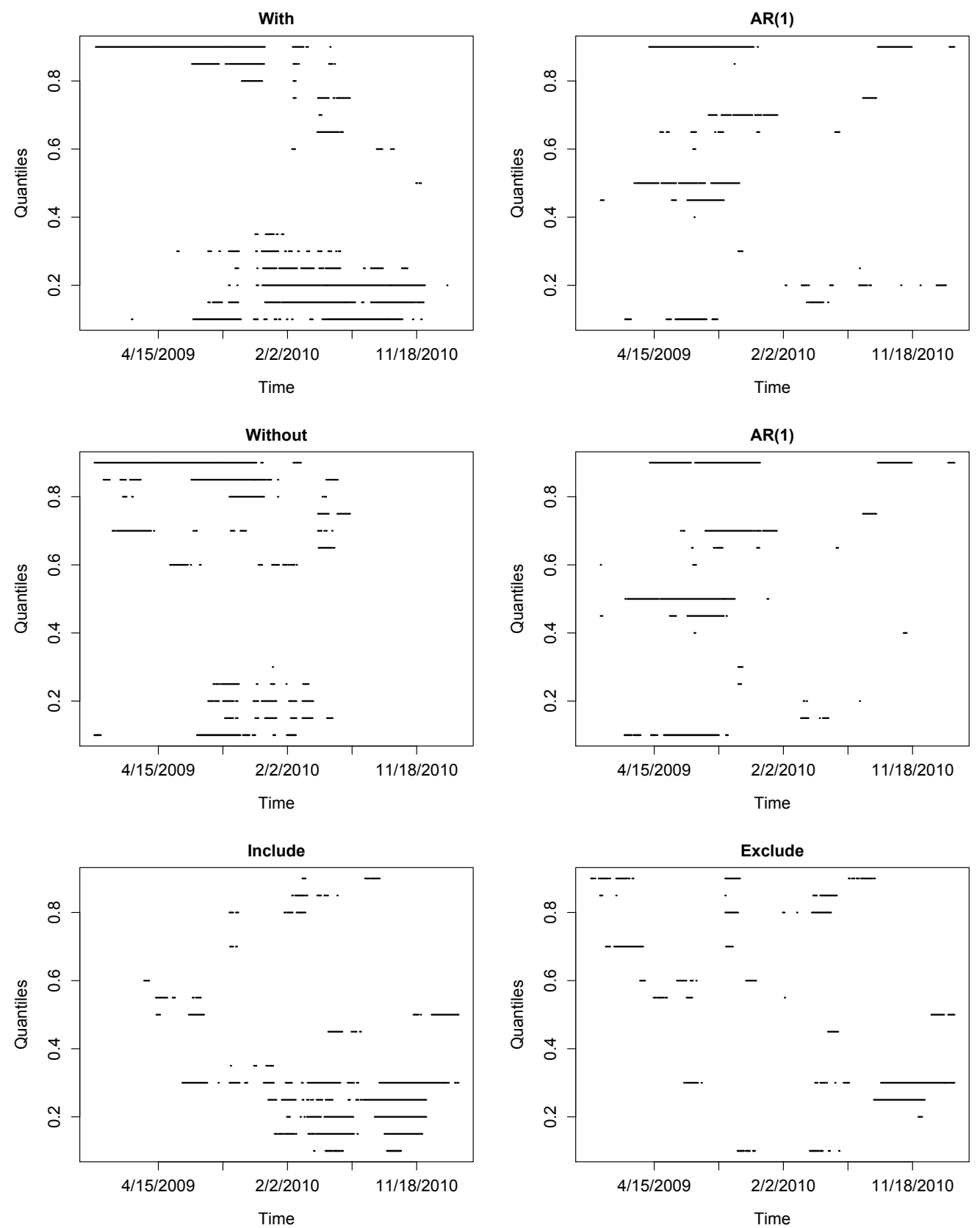

Note: Every dot represents a p-value smaller than 5\%. p-values are based on 1,000 bootstrap simulations. Results are based on the extended out-of-sample period, where the Fair-Shiller regressions are estimated on a rolling estimation window of length 250 days. With/AR(1) = results of Fair-Shiller regressions when the boosted model includes the realized moments in the list of potential predictor variables and a boosted AR(1) model is the benchmark model. Without/AR(1) = results of Fair-Shiller regressions when the boosted model excludes the realized moments in the list of potential predictor variables and a boosted $\operatorname{AR}(1)$ model is the benchmark model. Include/Exclude $=$ results of Fair-Shiller regressions include forecasts from the boosted models that include/exclude the realized moments in the list of potential predictor variables. 
Figure 5: Rolling Fair-Shiller Regressionens (Ten-Days-Ahead Returns)
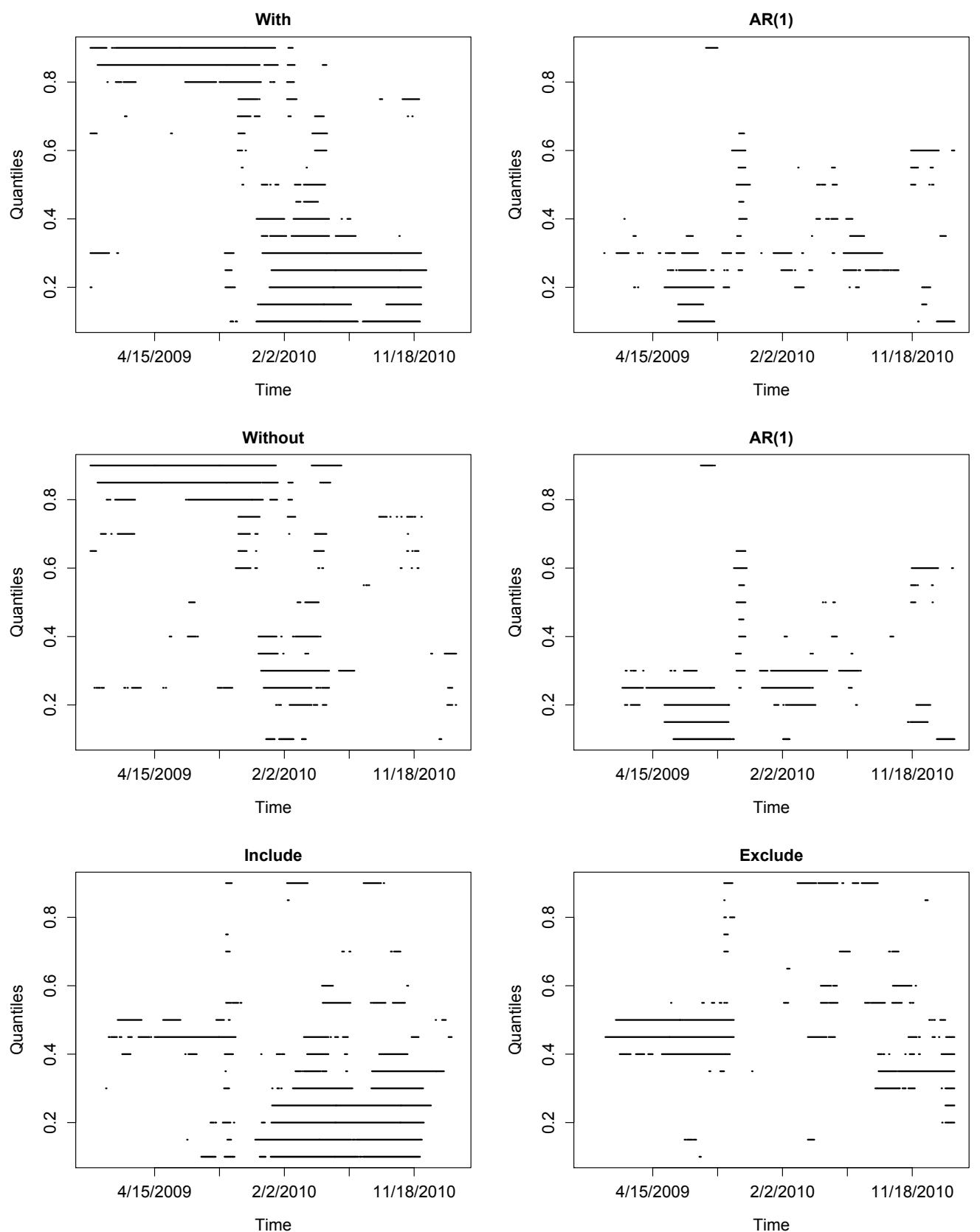

Note: Every dot represents a p-value smaller than 5\%. p-values are based on 1,000 bootstrap simulations. Results are based on the extended out-of-sample period, where the Fair-Shiller regressions are estimated on a rolling estimation window of length 250 days. With/AR(1) = results of Fair-Shiller regressions when the boosted model includes the realized moments in the list of potential predictor variables and a boosted AR(1) model is the benchmark model. Without/AR(1) = results of Fair-Shiller regressions when the boosted model excludes the realized moments in the list of potential predictor variables and a boosted $\operatorname{AR}(1)$ model is the benchmark model. Include/Exclude $=$ results of Fair-Shiller regressions include forecasts from the boosted models that include/exclude the realized moments in the list of potential predictor variables. 


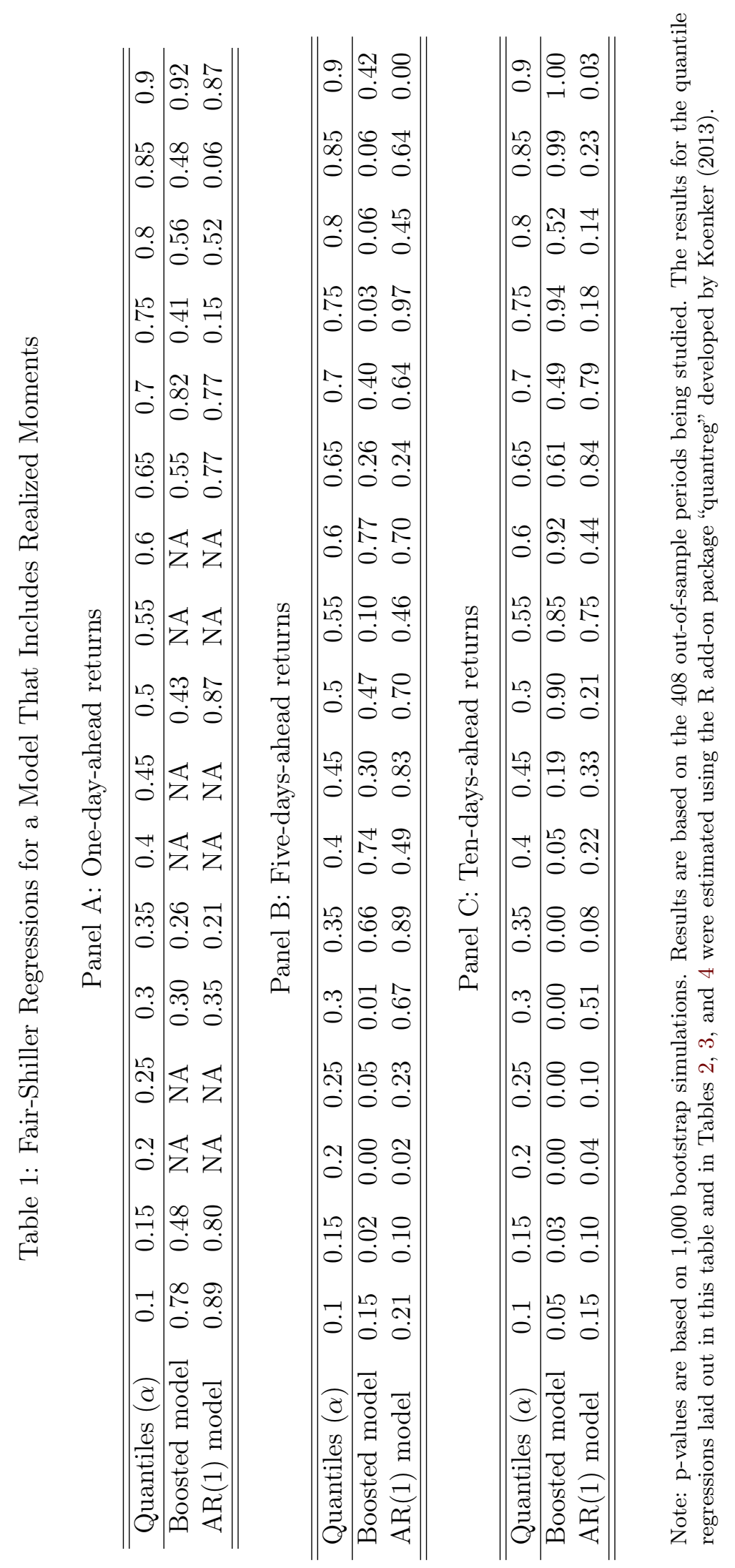




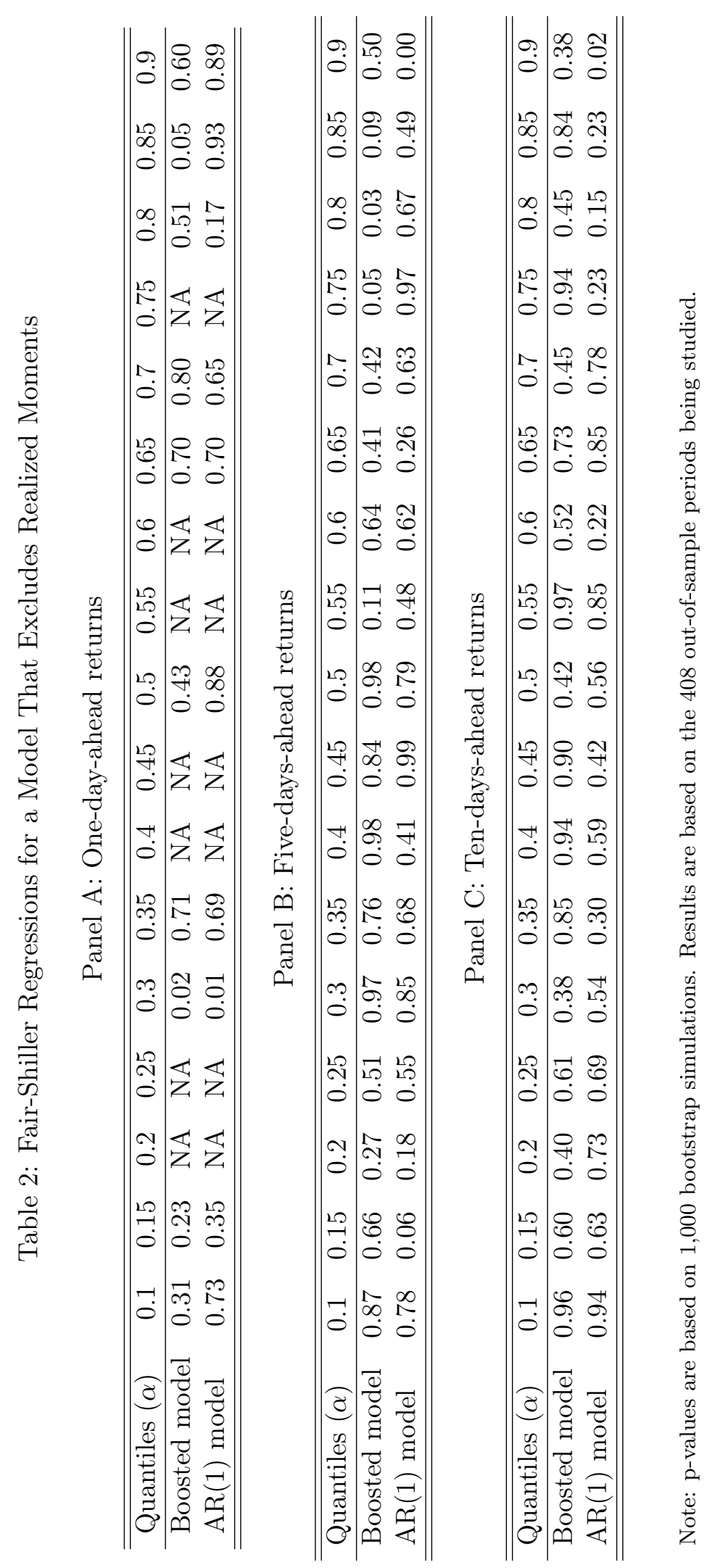




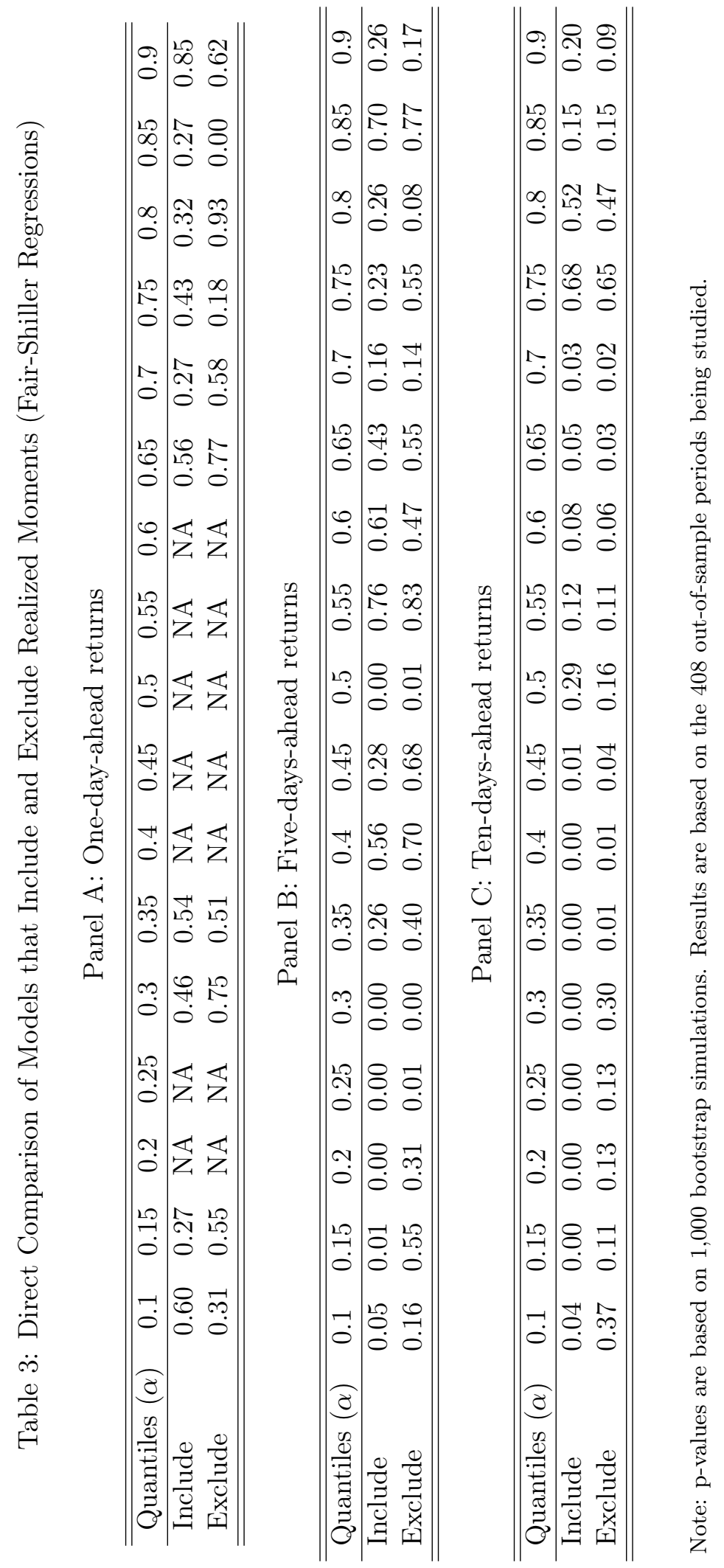




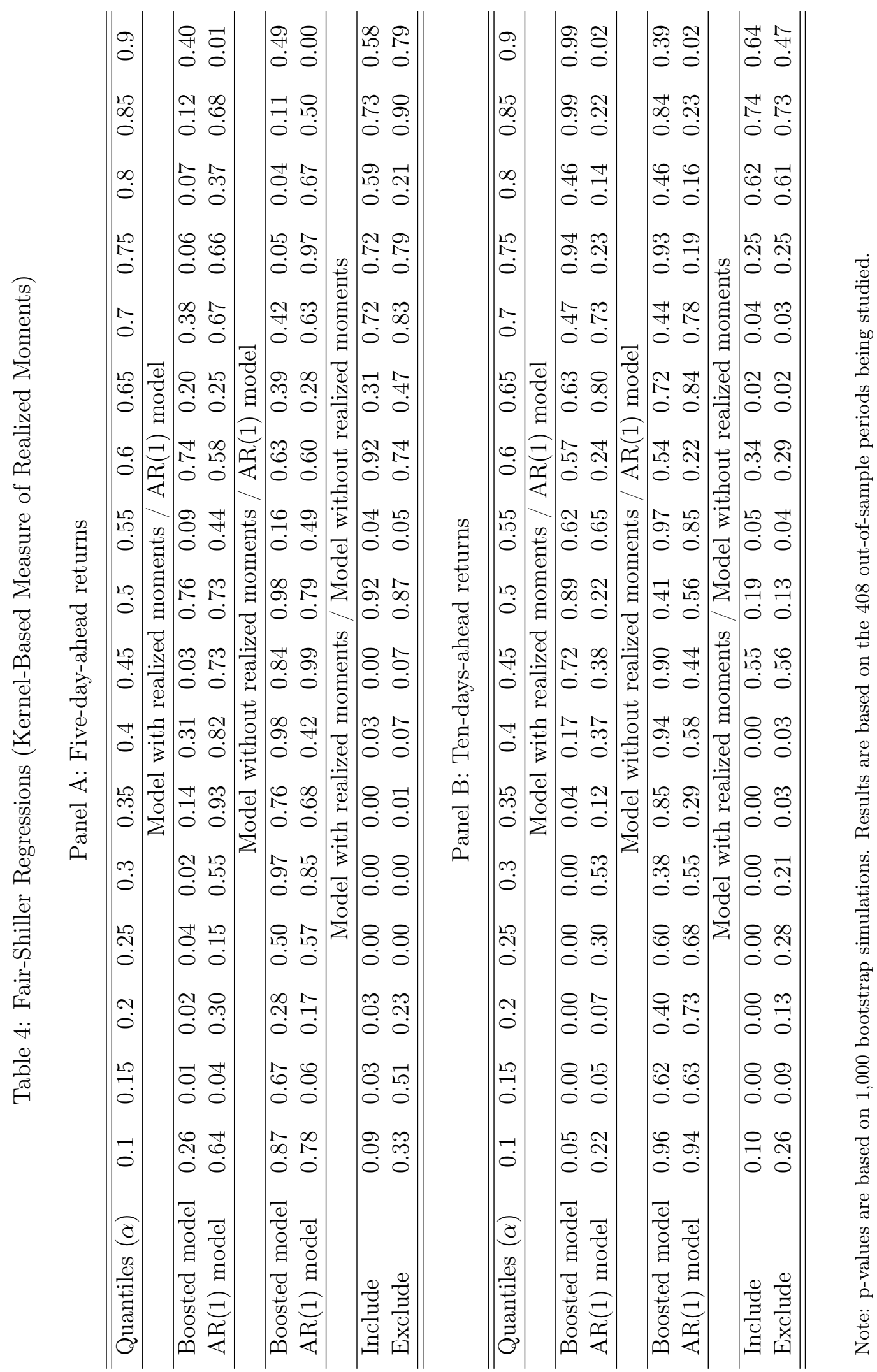




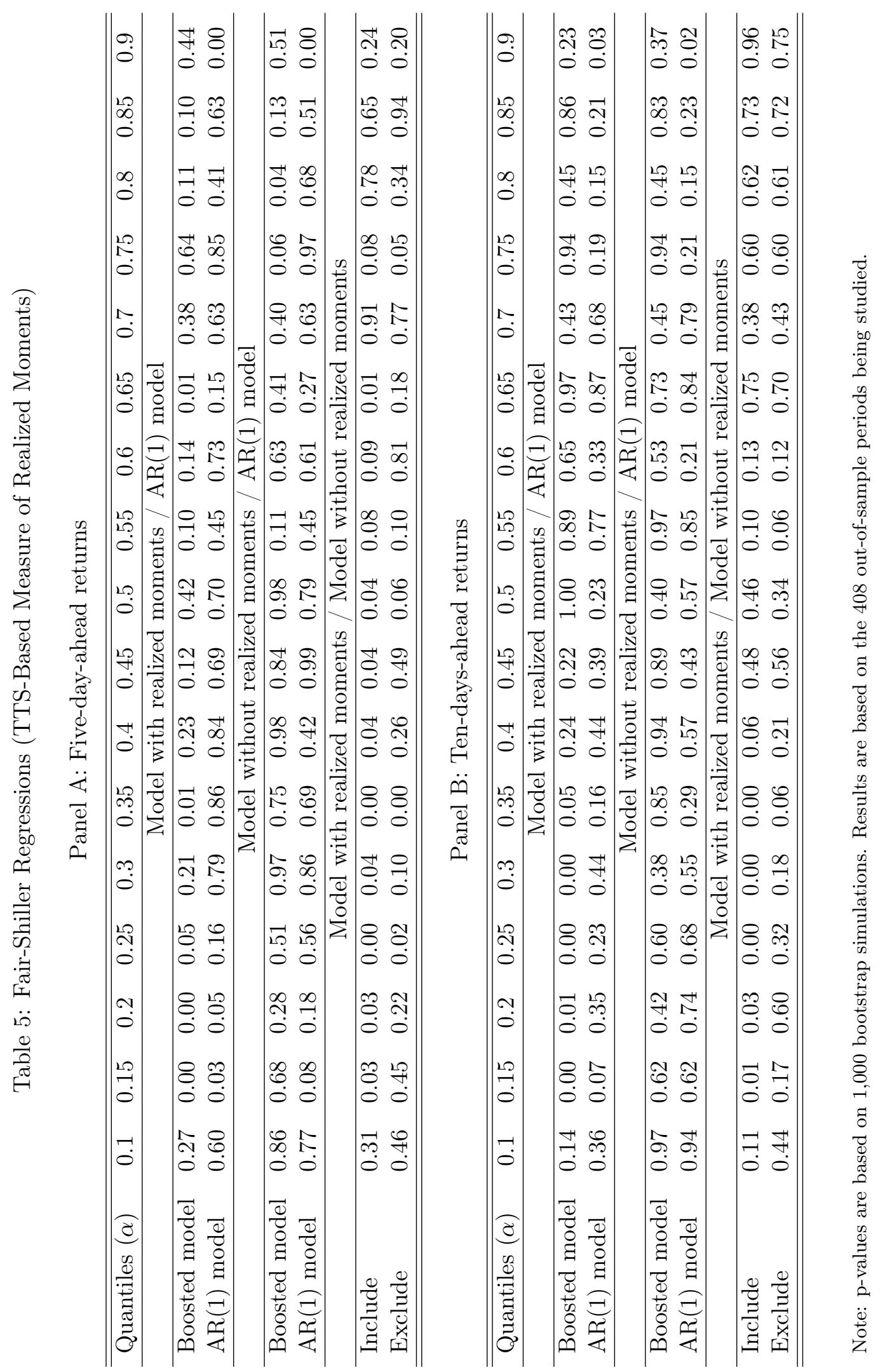

